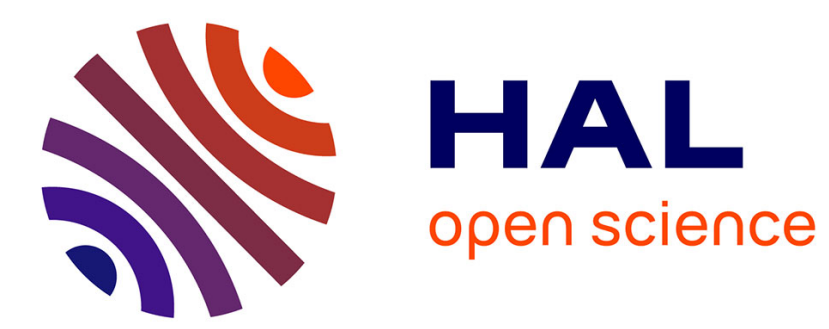

\title{
Interaction-free delayed-choice scenario
}

\author{
D.L. L Khokhlov
}

\section{To cite this version:}

D.L. L Khokhlov. Interaction-free delayed-choice scenario. Optik, 2022, 250, pp.168174. 10.1016/j.ijleo.2021.168174 . hal-03494474

\section{HAL Id: hal-03494474 https://hal.science/hal-03494474}

Submitted on 21 Dec 2021

HAL is a multi-disciplinary open access archive for the deposit and dissemination of scientific research documents, whether they are published or not. The documents may come from teaching and research institutions in France or abroad, or from public or private research centers.
L'archive ouverte pluridisciplinaire HAL, est destinée au dépôt et à la diffusion de documents scientifiques de niveau recherche, publiés ou non, émanant des établissements d'enseignement et de recherche français ou étrangers, des laboratoires publics ou privés. 


\title{
Interaction-free delayed-choice scenario
}

\author{
D.L. Khokhlov \\ Sumy State University (Retired), Ukraine
}

\begin{abstract}
The fully quantum description of the two path interference of a single photon in the Mach-Zehnder interferometer is presented. The interaction-free delayed-choice scenario in the Mach-Zehnder interferometer is proposed to test the fully quantum description of the two path interference. Several schemes of the arrangements designed to realize the interaction-free delayed-choice scenario are considered.
\end{abstract}

Key words: delayed-choice experiment, interaction-free experiment, Mach-Zehnder interferometer

\section{Introduction}

The two-path interferometer is a typical apparatus to investigate wave-particle duality of a single particle. One may study the behaviour of a single photon in the Mach-Zehnder interferometer, see Fig. 1. It works as follows. A single photon enters the interferometer through the first non-polarizing 50/50 beam splitter. The state of the photon is split in two components, passing through the legs $a$ and $b$, and then coupling at the second non-polarizing 50/50 beam splitter. The interference of the two components takes place at the second beam splitter due to the difference of the number of reflections on the legs $a$ and $b$. Provided the length of the legs $a$ and $b$ is the same, the photon leaves the interferometer through the bright port of the second beam splitter, with the probability $100 \%$, and through the dark port of the second beam splitter, with zero probability. One may influence the interference, adding a phase with a phase shifter on one of the legs.

Making the projective measurement on the leg $a$ or $b$, one may ascertain which path the photon took. In this case, the interference at the second beam splitter will be destroyed. Which way information and interference represent the

Email address: dlkhokhl@rambler.ru (D.L. Khokhlov). 


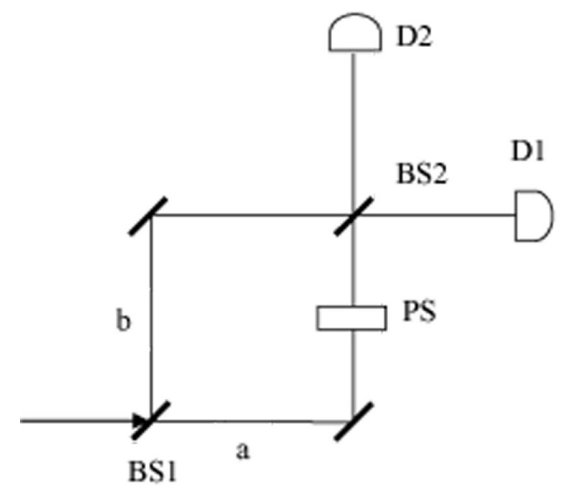

Fig. 1. Mach-Zehnder interferometer. BS: beam splitter, PS: phase shifter, D: detector.

particle and wave properties of the photon respectively. The Bohr's complementarity principle [1] postulates incompatibility of which way information and interference. It is worth to emphasize that the principle holds for the same configuration.

Elitzur and Vaidman [2] considered the concept of interaction-free measurement in the Mach-Zehnder interferometer. When an object blocks one of the paths, $a$ or $b$, the photon passing through the unblocked leg leaves the interferometer through the bright and dark ports, with equal probability $50 \%$. The interaction-free measurement allows to ascertain the presence or absence of the object on one of the legs indirectly. The experimental realization of the interaction-free measurement was reported in [3].

Wheeler [4] considered the concept of the delayed-choice experiment in the Mach-Zehnder interferometer. Just before the photon reaches the exit beam splitter, one may randomly replace the exit beam splitter by the which way detector. That is, one may observe interference or obtain which way information at will. The Wheeler's idea was realized in several experiments, e.g. [5]. Authors of [6] proposed the quantum version of the delayed-choice experiment, replacing the exit beam splitter by the element in a superposition of being present or being absent. The idea of the quantum delayed-choice experiment was implemented in several experiments, e.g. [7]. The discussion of the realized delayed-choice experiments can be found in [8]. To this end, the version of the delayed-choice experiment in the classical configuration was proposed in [9].

The standard formalism of quantum mechanics [10] is semi-classical. It considers time not as a quantum operator but as a classical parameter, e.g. [11] and references therein. Several approaches to the quantization of time were under discussion [12], see also [13]. In what follows, we shall present the fully quantum description of the two path interference of a single photon, introducing the spatial and temporal states of the photon. We shall consider the interaction-free delayed-choice scenario allowing to test the fully quantum de- 
scription of the two path interference. We shall consider several schemes of the arrangements based on the Mach-Zehnder interferometer and polarizing Mach-Zehnder interferometer designed to realize the interaction-free delayedchoice scenario.

\section{Spatial and temporal states of the photon}

Consider an approach to describe the two-path interference of a single photon in the Mach-Zehnder interferometer in the fully quantum manner. Suppose that one can develop the formalism of quantum mechanics, including the operators of spacial coordinate and time. The assumed operator of time is orthogonal to the operator of space coordinate. In this case, the spatial and temporal states are incompatible. Introduce the spatial state of the photon with the momentum $p$ as

$$
|\psi(r)\rangle=e^{i p r}
$$

and the temporal state of the photon with the energy $E$ as

$$
|\psi(t)\rangle=e^{i E t}
$$

The behaviour of the spatial and temporal states of the photon is restricted by the Heisenberg uncertainty relations in the momentum-space and in the energy-time domains respectively [14].

Due to the Heisenberg momentum-space uncertainty relation, the spatial state of the photon can be defined at a given time in an infinite space (in the region limited by the experimental conditions). Although the spatial state of the photon is defined at a given time, it is established with a relativistic time delay. The spatial state of the photon in the Mach-Zehnder interferometer can be defined at the time when the photon reaches the exit beam splitter in the space of the interferometer. The spatial state of the photon in the Mach-Zehnder interferometer can be cast in the form

$$
|\psi(r)\rangle=\left(\left|\psi_{a}\right\rangle+\left|\psi_{b}\right\rangle\right) / \sqrt{2}
$$

Here, $\left|\psi_{a}\right\rangle$ is the spatial state defined along the leg $a$ from the first to the second beam splitter, $\left|\psi_{b}\right\rangle$ is the spatial state defined along the leg $b$ from the first to the second beam splitter. The state eq. (3) can describe the interference of the paths $a$ and $b$ at the time when the photon reaches the exit beam splitter but it is not suitable to describe the travel of the photon through the interferometer. 
To this end, the approach developed in $[15,16]$ treats the superposition of the paths $a$ and $b$ as a state for controlling the apparatus.

Due to the Heisenberg energy-time uncertainty relation, the temporal state of the photon can be defined in a given point of space in an infinite time (in the interval of time limited by the experimental conditions). The temporal state of the photon in the Mach-Zehnder interferometer can be defined in the point of space associated with the photon as a single particle in the interval of time corresponding to the travel of the photon through the interferometer. Because of the probabilistic work of the beam splitters, the way the photon took in the interferometer is unknown. The temporal state of the photon in the Mach-Zehnder interferometer can be cast in the form

$$
|\psi(t)\rangle=\left(|t\rangle-\left|t_{0}\right\rangle\right)\left(\left|1_{a}\right\rangle\left|0_{b}\right\rangle+\left|0_{a}\right\rangle\left|1_{b}\right\rangle\right) / \sqrt{2}
$$

where $\left|t_{0}\right\rangle$ is the initial time, $|1\rangle$ and $|0\rangle$ stand for the single particle and vacuum states respectively. The state eq. (4) allows to define the position of the photon in the interferometer at any time $t$, on the leg $a$ or $b$. It can describe the travel of the photon through the interferometer but is not suitable to describe the interference of the paths $a$ and $b$.

The spatial state of the photon eq. (3) and the temporal state of the photon eq. (4) give the fully quantum description of the two-path interference of a single photon in the Mach-Zehnder interferometer. The temporal state of the photon eq. (4) can describe the travel of the photon through the interferometer. The spatial state of the photon eq. (3) can describe the interference of the paths $a$ and $b$. The temporal state of the photon is associated with the particle behaviour of the photon while the spatial state of the photon is associated with the wave behaviour of the photon.

In the semi-classical formalism of quantum mechanics [10], the spatial components of the photon propagate along the legs $a$ and $b$ in time, accumulating the phase in time. The interference of the paths $a$ and $b$ is defined by the difference of the phase in time between the legs $a$ and $b$. In the fully quantum approach under consideration, the interference of the paths $a$ and $b$ is defined by the difference of the phase in space between the legs $a$ and $b$ at the time the interference takes place. We shall consider a scenario which may distinguish the fully quantum description of the two path interference from the semi-classical one.

One may change the configuration of the interferometer before the photon reaches the exit beam splitter thus realizing the delayed-choice scenario. We shall consider the delayed-choice scenario in which the part of the interferometer is changed behind the photon. In this case, the photon cannot pass through the part of the interferometer which has been changed. Such a delayed-choice 
scenario is interaction-free. The change of the configuration of the interferometer gives rise to the change of the state of the photon in the space of the interferometer that may influence the interference of the photon at the exit beam splitter. The interaction-free delayed-choice scenario allows to perform a test to distinguish between the fully quantum and the semi-classical descriptions of the two path interference.

\section{Schemes of the arrangements}

Consider several schemes of the arrangements designed to realize the interactionfree delayed-choice scenario. Consider the scheme of the arrangement depicted in Fig. 1. It is a Mach-Zehnder interferometer, with a phase shifter on the leg $a$, either a phase delay element or a half wave plate.

Let a single photon enter the interferometer. Let a phase delay element be placed on the leg $a$. Before the photon reaches the phase delay element, set zero phase shift. The component of the temporal state of the photon eq. (4) on the leg $a$ passes through the phase delay element, with no phase shift. After the photon passes the phase delay element and before it reaches the exit beam splitter, set a phase shift of $\pi / 2$. At the time when the photon reaches the exit beam splitter, the component of the spatial state of the photon eq. (3) on the leg $a$ has a phase shift of $\pi / 2$. Therefore, the components of the state eq. (3) on the legs $a$ and $b$ are completely distinguishable that destroys the interference. As a result, the photon may leave the interferometer through the bright and dark ports, with equal probability $50 \%$.

Now, set a phase shift of $\pi / 2$ before the photon reaches the phase delay element. The component of the temporal state of the photon eq. (4) on the leg $a$ passes through the phase delay element, acquiring a phase shift of $\pi / 2$. After the photon passes the phase delay element and before it reaches the exit beam splitter, set zero phase shift. At the time when the photon reaches the exit beam splitter, the component of the spatial state of the photon eq. (3) on the leg $a$ has zero phase shift. There is no phase difference between the legs $a$ and $b$, i.e. the components of the spatial state of the photon eq. (3) on the legs $a$ and $b$ are indistinguishable, and the interference of the photon at the second beam splitter takes place. As a result, the photon leaves the interferometer through the bright port, with the probability $100 \%$.

Let a single photon with the horizontal polarization $H$ enter the interferometer. Let a half wave plate be placed on the leg $a$. Before the photon reaches the half wave plate, switch off the half wave plate. The component of the temporal state of the photon eq. (4) on the leg a passes through the half wave plate, without changing its polarization. After the photon passes the half wave plate 
and before it reaches the exit beam splitter, switch on the half wave plate. At the time when the photon reaches the exit beam splitter, the component of the spatial state of the photon eq. (3) on the leg $a$ has the vertical polarization $V$ while the component of the spatial state of the photon eq. (3) on the leg $b$ remains in the horizontal polarization $H$. Therefore, the components of the state eq. (3) on the legs $a$ and $b$ are completely distinguishable that destroys the interference. As a result, the photon may leave the interferometer through the bright and dark ports, with equal probability $50 \%$.

Now, switch on the half wave plate before the photon reaches the half wave plate. The component of the temporal state of the photon eq. (4) on the leg $a$ passes through the half wave plate, changing its polarization to the vertical one $V$. After the photon passes the half wave plate and before it reaches the exit beam splitter, switch off the half wave plate. At the time when the photon reaches the exit beam splitter, the component of the spatial state of the photon eq. (3) on the leg $a$ has the horizontal polarization $H$ the same as the component of the spatial state of the photon eq. (3) on the leg $b$. Therefore, the components of the spatial state of the photon eq. (3) on the legs $a$ and $b$ are indistinguishable, and the interference of the photon at the second beam splitter takes place. As a result, the photon leaves the interferometer through the bright port, with the probability $100 \%$.

Consider the scheme of the arrangement depicted in Fig. 2. It is a MachZehnder interferometer with two exit beam splitters. There are two nested Mach-Zehnder interferometers, delimited by the beam splitters BS2 and BS3 on the leg $a$, and by the beam splitters BS4 and BS5 on the leg $b$. After the beam splitter BS3, the leg $a$ is split into the legs $a_{1}$ and $a_{2}$. After the beam splitter BS5, the leg $b$ is split into the legs $b_{1}$ and $b_{2}$. The legs $a_{1}$ and $b_{1}$ go to the first exit beam splitter. The legs $a_{2}$ and $b_{2}$ go to the second exit beam splitter. Suppose that the length of the legs $a$ and $b$ is the same, and the length of the legs $a_{1}, a_{2}, b_{1}$ and $b_{2}$ is the same. The nested Mach-Zehnder interferometers serve to switch from the two-path to one-path regime by means of the phase shifters. In the two-path regime, the legs $a_{1}, a_{2}, b_{1}$ and $b_{2}$ are open. In the one-path regime, the legs $a_{1}$ and $b_{2}$ are open while the legs $a_{2}$ and $b_{1}$ are closed.

Let a single photon enter the interferometer. The photon in the temporal state eq. (4) passes through the leg $a$ or $b$. Let the legs $a_{1}$ and $b_{2}$ be open and the legs $a_{2}$ and $b_{1}$ be closed. Then, the photon passes through the leg $a_{1}$ or $b_{2}$. The temporal state of the photon takes the form

$$
|\psi(t)\rangle=\left(|t\rangle-\left|t_{0}\right\rangle\right)\left(\left|1_{a_{1}}\right\rangle\left|0_{b_{2}}\right\rangle+\left|0_{a_{1}}\right\rangle\left|1_{b_{2}}\right\rangle\right) / \sqrt{2}
$$

After the photon leaves one of the nested Mach-Zehnder interferometers and before it reaches one of the exit beam splitters, open the legs $a_{2}$ and $b_{1}$. At the time when the photon reaches the first or the second exit beam splitter, 


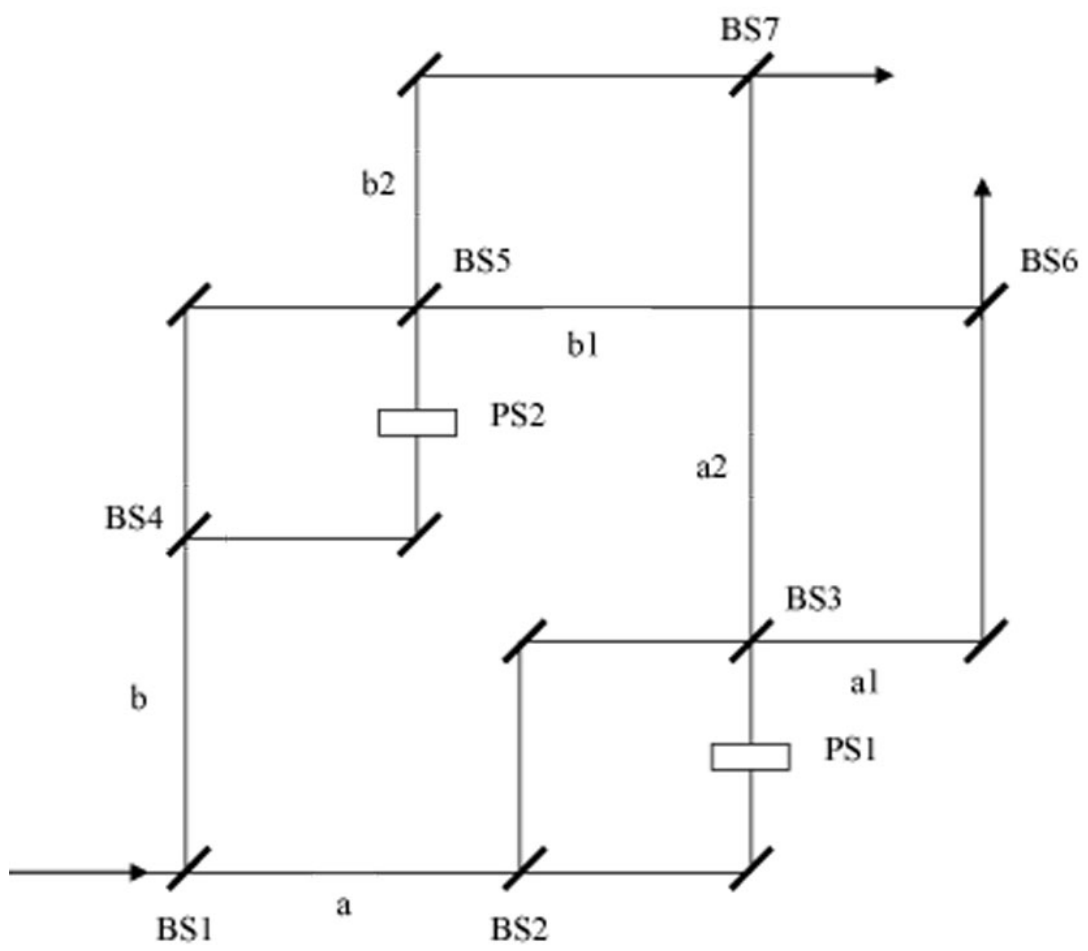

Fig. 2. Mach-Zehnder interferometer with two exit beam splitters. BS: beam splitter, PS: phase shifter.

the spatial state of the photon is given by

$$
|\psi(r)\rangle=\left(\left|\psi_{a_{1}}\right\rangle+\left|\psi_{b_{1}}\right\rangle\right) / 2+\left(\left|\psi_{a_{2}}\right\rangle+\left|\psi_{b_{2}}\right\rangle\right) / 2 \text {. }
$$

The components of the state eq. (6) on the legs $a_{1}$ and $b_{1}$ are indistinguishable at the first exit beam splitter. The components of the state eq. (6) on the legs $a_{2}$ and $b_{2}$ are indistinguishable at the second exit beam splitter. Therefore, the interference takes place at one of the exit beam splitters, and the photon leaves the interferometer through the bright port of one of the exit beam splitters. Detecting the photon after the first or the second exit beam splitter, one may ascertain which path the photon took, $a_{1}$ or $b_{2}$.

The interaction-free delayed-choice scenario in the Mach-Zehnder interferometer with two exit beam splitters allows to have both which way information and interference. This is arranged in the different configurations, in accordance with the Bohr's complementarity principle. To this end, scenarios to obtain which way information without destroying interference were considered in [17] using weak measurement and in [18] using quantum non-demolition measurement.

Consider the scheme of the arrangement depicted in Fig. 3. It is a polarizing Mach-Zehnder interferometer with two exit beam splitters similar to that de- 
picted in Fig. 2, with the polarizing beam splitters instead of the entrance and exit non-polarizing ones. Let a single photon in the polarization state $|H+V\rangle$ enter the interferometer. After the entrance beam splitter, the photon goes through the interferometer in the temporal state coupled to the polarization state

$$
|\psi(t)\rangle=\left(|t\rangle-\left|t_{0}\right\rangle\right)\left(\left|1 H_{a}\right\rangle\left|0 V_{b}\right\rangle+\left|0 H_{a}\right\rangle\left|1 V_{b}\right\rangle\right) / \sqrt{2} .
$$

Let the legs $a_{1}$ and $b_{2}$ be open and the legs $a_{2}$ and $b_{1}$ be closed. Then, the photon passes through the leg $a_{1}$ or $b_{2}$. The temporal state of the photon coupled to the polarization state takes the form

$$
|\psi(t)\rangle=\left(|t\rangle-\left|t_{0}\right\rangle\right)\left(\left|1 H_{a_{1}}\right\rangle\left|0 V_{b_{2}}\right\rangle+\left|0 H_{a_{1}}\right\rangle\left|1 V_{b_{2}}\right\rangle\right) / \sqrt{2}
$$

After the photon leaves one of the nested Mach-Zehnder interferometers and before it reaches one of the exit beam splitters, open the legs $a_{2}$ and $b_{1}$. At the time when the photon reaches the first or the second exit beam splitter, the spatial state of the photon coupled to the polarization state is given by

$$
|\psi(r)\rangle=\left(\left|H_{a_{1}}\right\rangle+\left|V_{b_{1}}\right\rangle\right) / 2+\left(\left|H_{a_{2}}\right\rangle+\left|V_{b_{2}}\right\rangle\right) / 2 .
$$

The interference in the polarization space takes place at one of the exit beam splitters. As a result, the photon leaves one of the exit beam splitters in the polarization state $|H+V\rangle$. The polarization state of the photon is the same before and after the interferometer.

The polarizing Mach-Zehnder interferometer with two exit beam splitters allows to obtain which way information, keeping the initial polarization state of the photon. Multiple passage of the photon through the interferometer may be used to collect statistics to discriminate between the different polarization states of the photon. To this end, a scenario to obtain which way information in the modified polarizing Mach-Zehnder interferometer, keeping the initial polarization state of the photon, was considered in [19] using weak measurement. The scheme of the arrangement, consisting of several modified polarizing Mach-Zehnder interferometers [19], was suggested in [20] to perform an attack on the protocol BB84. Following [20], one may apply the polarizing Mach-Zehnder interferometer with two exit beam splitters to perform an attack on the protocol BB84. 


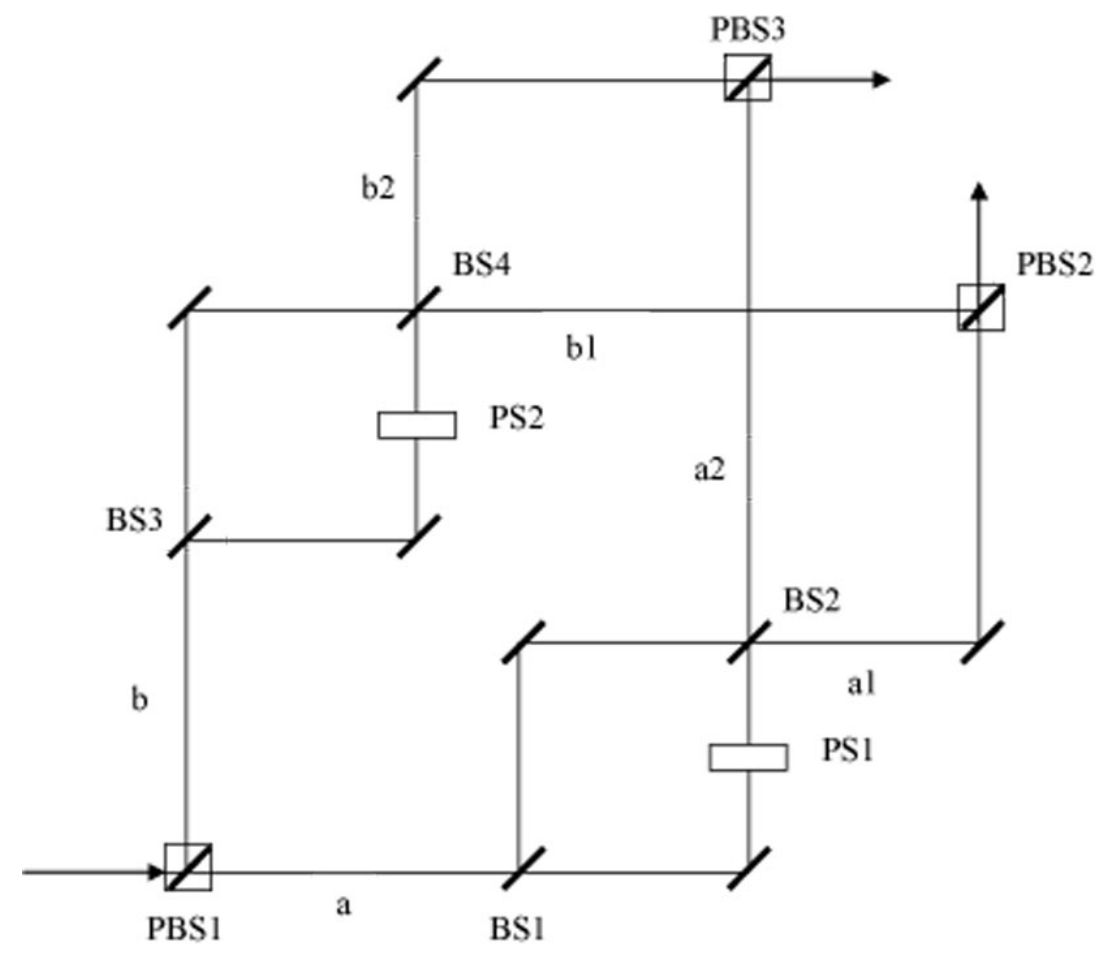

Fig. 3. Polarizing Mach-Zehnder interferometer with two exit beam splitters. PBS: polarizing beam splitter, BS: beam splitter, PS: phase shifter.

\section{Conclusion}

We have presented the fully quantum description of the two path interference of a single photon in the Mach-Zehnder interferometer, introducing the spatial and temporal states of the photon. The temporal state of the photon can describe the travel of the photon through the interferometer, and the spatial state of the photon can describe the two path interference. In this approach, the interference of the paths $a$ and $b$ is defined by the difference of the phase in space between the legs $a$ and $b$ at the time the interference takes place.

The interaction-free delayed-choice scenario in the Mach-Zehnder interferometer has been proposed to test the fully quantum description of the two path interference. The part of the interferometer is changed behind the photon such that the photon cannot pass through this part. The change of the configuration of the interferometer gives rise to the change of the state of the photon in the space of the interferometer that influences the interference of the photon at the exit beam splitter. Several schemes of the arrangements designed to realize the interaction-free delayed-choice scenario have been considered.

The scheme of the arrangement based on the Mach-Zehnder interferometer has been considered. A phase shifter is placed on one of the legs, either a 
phase delay element or a half wave plate. After the photon passes the phase shifter and before it reaches the exit beam splitter, one may switch the phase shifter, adding a phase on one of the legs. This does not change the temporal state of the photon and does the spatial state of the photon that influences the interference of the photon at the exit beam splitter.

The scheme of the arrangement being a Mach-Zehnder interferometer with two exit beam splitters has been considered. After the entrance beam splitter, two legs are split into four legs which then couple at two exit beam splitters. One may close two of four legs such that the photon reaches one of the exit beam splitters through the definite leg. Before the photon reaches one of the exit beam splitters, one may open two closed legs. In this case, the interference takes place at one of the exit beam splitters. Thus, the Mach-Zehnder interferometer with two exit beam splitters allows to have both which way information and interference. This is arranged in the different configurations, in accordance with the Bohr's complementarity principle.

The scheme of the arrangement being a polarizing Mach-Zehnder interferometer with two exit beam splitters has been considered. It works like that in the previous scheme. The arrangement allows to obtain which way information, keeping the initial polarization state of the photon. Multiple passage of the photon through the interferometer allows to discriminate between the different polarization states of the photon that may be used to perform an attack on the protocol BB84.

\section{References}

[1] N. Bohr, Atomic Physics and Human Knowledge, Science Editions, New York, 1961.

[2] A.C. Elitzur, and L. Vaidman, Quantum mechanical interaction-free measurements, Found. Phys. 23 (1993) 987-997.

[3] P. Kwiat, H. Weinfurter, T. Herzog, A. Zeilinger, and M.A. Kasevich, Interaction-free measurement, Phys. Rev. Lett. 74 (1995) 4763-4766.

[4] J.A. Wheeler, Law without law, in J.A. Wheeler, and W.H. Zurek (Eds.), Quantum Theory and Measurement, Princeton University Press, 1984.

[5] V. Jacques, E. Wu, F. Grosshans, F. Treussart, P. Grangier, A. Aspect, and J.-F. Roch, Experimental realization of Wheeler's delayed-choice gedanken experiment, Science 315 (2007) 966-968.

[6] R. Ionicioiu, and D.R. Terno, Proposal for a quantum delayed-choice experiment, Phys. Rev. Lett. 107 (2011) 230406. 
[7] J.-S. Tang, Y.-L. Li, X.-Y. Xu, G.-Y. Xiang, C.-F. Li, and G.-C. Guo, Realization of quantum Wheeler's delayed-choice experiment, Nature Photon. 6 (2012) 602-606.

[8] X.-S. Ma, J. Kofler, A. Zeilinger, Delayed-choice gedanken experiments and their realizations, Rev. Mod. Phys. 88 (2016) 015005.

[9] D.L. Khokhlov, Two-slit experiment with two screens, Optik 179 (2019) 557559 .

[10] J. von Neumann, Mathematical Foundations of Quantum Mechanics, Princeton University Press, 1955.

[11] H. Nikolić, Quantum mechanics: Myths and facts, Found. Phys. 37 (2007) 15631611.

[12] J.G. Muga, R. Sala Mayato, and I.L. Egusquiza (Eds.), Time in quantum mechanics, 2nd ed., Springer-Verlag, Berlin, 2007.

[13] D.L. Khokhlov, Wave functions of the photon in space and time, in Z. Ezziane (Ed.), Contemporary Research in Quantum Systems, Nova Science Publishers, Hauppauge NY, 2014, p.p. 199-206.

[14] D.L. Khokhlov, Spatial and temporal wave functions of photon, Appl. Phys. Res. 2(2) (2010) 49-54.

[15] D.L. Khokhlov, Interaction of the classical particle and quantum apparatus, Int. J. Gen. Syst. 44 (2015) 106-110.

[16] D.L. Khokhlov, Interference of a single photon in the Mach-Zehnder interferometer, Quan. Inf. Rev. 7 (2019) 7-10.

[17] D.L. Khokhlov, Modification of the two-slit experiment with a single photon, Optik, 126 (2015) 5301-5303.

[18] D.L. Khokhlov, Quantum non-demolition measurement in the interferometer, Int. J. Phys. Res. 9 (2021) 7-10.

[19] D.L. Khokhlov, Scheme of the weak measurement of the polarization state of the photon, Optik 127 (2016) 4089-4091.

[20] D.L. Khokhlov, Scheme of the arrangement for attack on the protocol BB84, Optik 127 (2016) 7083-7087. 